\title{
INVOLUNTARY CONVERGENCE, EYESTRAIN AND SQUINT
}

\author{
BY \\ N. A. Stutterheim \\ JOHANNESBURG
}

In ophthalmology there are two schools of thought, the physiological and the physical or mechanical school. Thus Williamson-Noble ${ }^{1}$, dealing with my method of treating eyestrain, states: "curiously enough, Stutterheim designates the condition which his exercises are designed to improve as "involuntary convergence," whereas, as you will shortly see, it is the ability to perform the act voluntarily which is of real importance." He then describes how he has changed my technique of treatment into one with the rotary prism and the synoptophore, giving the "job" to the orthoptic trainer rather than doing it himself. This is the mechanical outlook.

On the other hand $Z$ eeman ${ }^{2}$, collaborator of Brouwer ${ }^{3}$ at the Institute for Brain Physiology, in a press note on my work "Eyestrain and Convergence", states concerning my method: "It aims at harmonic training and widening of the important function of convergence, which is correctly called " involuntary."

By Elschnig 5 convergence movements are called fusion movements. The "fusion movements" Elschnig refers to are the movements of convergence, as his description shows. He writes : "These movements are eye movements that alter the position of the eyes in such a way that the visual lines at every moment meet in the object of fixation. In normal binocular vision they occur unconsciously and involuntarily." (The italics are mine.)

The range of these movements Elschnig gives as the sum of the strongest abducing prism and the strongest adducing prism that can be overcome by binocular fixation of a very far point without diplopia occurring. The normal abduction range (negative convergence) Elschnig gives as about 8 dioptres of prism, the normal adduction range (convergence) he gives as about 50 dioptres of prism.

Duane $^{6}$ measures a (voluntary) convergence, which he defines in this way: "The convergence power in a given case is best measured by determining the closest point to which a fine object accurately fixed by both eyes can be brought without either eye diverging." This is the so-called convergence test of, for instance, the R.A.F. It is simply a test for the muscular strength of voluntary squinting, or binocular adduction, very useful for that purpose, but by no means a test for real convergence, as the following figures will show.

The range of normal convergence (or normal fusion movement according to the nomenclature of Elschnig (see also $\left({ }^{4}\right)$, p. 5), is 50 dioptres of prism. The equivalent of this is about 26 degrees, 
whereas, according to Duane's measurements, very low "convergence " is 32-36 degrees, of which the equivalent, viz., 60-68 dioptres is already considerably higher than normal convergence. Then there is low 36-44 degrees, medium 44-54 degrees, high 54-64 degrees, and very high "convergence" 64-76 degrees. The equivalents in prism dioptres of these degrees are respectively between the very low equivalent 60-68 dioptres, and very high equivalent 120-144 dioptres of prism. These figures unmistakably prove that the socalled convergence, the voluntary kind, is an eye movement of a very different nature from the real convergence which has to do with eyestrain. This latter convergence is tested at a very far point, and is treated with prisms in order to bring it to the normal power of 50 dioptres of prism. Moreover people with astheno-vergence, with a range of convergence power lower than 20, or even 10 dioptres, usually are able to stand the "convergence test" to medium, high and often even very high binocular adduction.

Methodically exciting the patellar reflex, some would perhaps call "exercises." Since, however, it is not physical but physiological work, the physiologist would prefer to call this "treatment." The word exercises should be reserved for gymnastics such as methodical training in ordinary kicking. It is true that this kicking is done with the same group of extensors as are used in the more delicate reflex movement induced by hitting the ligamentum patellae, but there is a difference between them for all that.

The person subjected to the patellar reflex treatment is well aware that he does not act voluntarily. And it is remarkable that, when giving kinetic treatment for astheno-vergence with the battery of prisms, as proposed by me, one meets with nothing but surprise on the part of intelligent patients at the behaviour of the two images. I have personally given kinetic treatment to nearly two thousand people, and am used to patients exclaiming: "Oh, what has happened, there is another image," and "now they have come together again," or " they split, it goes further .... now they are one again," or " there is another one, off the screen," or " they come nearer, but will not join," and similar expressions. If these people had used voluntary convergence, they would know it, and not use such expressions. When one asks them, they admit that they cannot help either the splitting or the coming together of the images. Their expressions indicate that they have had a visual experience of something beyond their control, as with the patellar reflex act.

Their remarks mean that they have noted the result of a visual reflex, not the performance of a voluntary act.

If patients, during "lessons" given by another technique than mine, get the impression that they converge voluntarily, I fully understand it. The person subjected to the patellar reflex treatment, if he is allowed or even invited to kick during the reflex 
movement, will not be conscious of the powerful but more delicate reflex action, but merely of the far coarser movement of kicking.

In regular kinetic treatment some voluntary activity may come later, after three weeks or so. By then the 50 dioptres range has usually been passed. It may happen then that, when the images drift too far apart for involuntary convergence to overcome it alone, voluntary effort may bring them closer together. This does occur, though not in the majority of cases, and I have always avoided drawing the patient's attention to this possibility. The final joining of the two images remains as always, an involuntary act.

In Williamson-Noble's modification of the technique used and described by me, the procedure has not merely been shortened, but the battery method has been rejected and substituted by treatment with the rotary prisms and the synoptophore. I have warned the readers of both my books against the use of such instruments in this therapy.

Williamson-Noble describes his five lessons very extensively and in great detail because he is out to prove that the convergence elicited is "essentially voluntary and not involuntary in character" 1.

The fact is that after reading his description of the five lessons, I cannot detect any proof as to whether the act of convergence produced by him is voluntary or involuntary. Here applies what I have written in "Eyestrain and Convergence," p. 26, where I describe my experience of the assistance voluntary binocular adduction can give to convergence in difficult circumstances, and state: "These facts are at the bottom of the erroneous impression that there is such a thing as voluntary convergence."

It is regrettable that this more recent and more complete work on the subject ${ }^{4}$ has been entirely ignored in the lecture.

The process of treatment as given by me is called long and tedious, and the "job" is considered one for the orthoptic trainer rather than the ophthalmic surgeon. This is a matter not of taste but of outlook. I should never allow such a delicate powerful therapy out of the hands of the person with knowledge of physiology into those of a non-medical person. As it is, we already have grave reasons to object to refracting and eye-mirroring opticians, prescribing glasses to the suffering public. Moreover, Williamson-Noble's five lessons do not do justice to the underlying physiology of the problem.

For the sake of shortness, and, it seems, for economical reasons as well, the kinetic treatment of Williamson-Noble's five lessons is given forcefully, somewhat crudely even. He himself says: "they are usually made worse by the first two lessons, and it is well to warn them of this." Yet, the good results in even his selected 117 cases, treated by his trainer, are high ( 89 cures, 15 improvements), even seem to him "so unwarrantably high."

My good results, not in selected but in all cases of eyestrain which 
come under my care, give a higher percentage. And although I am glad to note that the method, even when applied in so crude a fashion, can give such good results, yet I should like to warn my colleagues not to endanger this proven asset of scientific and practical ophthalmology by haphazard techniques. A rotary prism is an excellent instrument, and so are the synoptiscope and the synoptophore, but I wish to repeat my warning against the use of these instruments in this treatment because of the obvious danger of undue mechanization.

Starting with the work of Donders and his contemporaries, ophthalmology has made astonishing progress, so-much-so that it gives the impression of a rounded off department of medicine, complete at least where its main issues are concerned. And so it is, when one contemplates the technical, optical and surgical aspects. Its achievements may well be sufficient to satisfy the ambition and claim the devotion of many a great medical mind. Nevertheless ophthalmology suffers from onesidedness. It has largely fallen into the hands of predominantly mechanically minded people. Physiology has become the Cinderella.

The enormous physical (surgical and optical) advance fascinates the adepts, and finds recruits almost entirely amongst mechanically minded students of medicine. As a result, new physiological conceptions are not grasped. Terms of method have replaced terms of function. Thus, when I drew attention to the functional unit whose insufficiency is responsible for most eyestrain, the new conception was overlooked, but the methods evolving out of it were taken up. This is the mechanical outlook. The newly revealed reflex convergence is constantly confused with voluntary adduction, and kinetic treatment, consequently is misnamed "exercises."

Surprise is general that the public are not placidly content with our method of refracting and its result, viz. : prescription of the most excellent glasses. Is there irony hidden in the remark" : in some cases it enables patients to discard their glasses, and this, in an age when every one is clamouring for exercises instead of glasses, may be of value?" This shows that we ophthalmologists are no longer ahead of our public. We do not try to help them radically. We should give them functional improvement first, and then, if really necessary, glasses. We show a misplaced contempt of the best, the most desirable part of our practice, those sensible people and intelligent parents who expect understanding of their complaints, but are only offered glasses.

It has been said that heresies were the unpaid debts of the church, and in a press note $e^{7}$ on my latest monograph, the reviewer has, not unkindly, called me a heretic. He writes: "It was only when the ideas promulgated in generations of textbooks were questioned by the so-called heretics of their day, that progress really became marked." 
Let us not overlook the signs of the times. Ophthalmology is losing ground-not, of course, where surgery or physical optics are concerned, but in other essential things. We have not kept faith with the public at large, and this is realized by our thinking professional colleagues.

Tedium is a matter of outlook. Some find physiological obstetrics tedious, and anything but forcipal interference a job for the midwife. Yet this outlook, if general, would bring about high infant mortality and high maternal morbidity.

With the battery of prisms, I have given kinetic treatment to nearly two thousand eyestrain patients. I have never found the procedure tedious, and am looking forward to the opportunity of curing another four thousand. Any ophthalmologist, who is not quite devoid of physiological outlook, can develop this new interest.

The advantages over our present-day chief occupation, refracting, followed by the unavoidable prescription of glasses, are considerable. One can nearly always assure one's patient that he has no anatomical or physical defect or weakness, and that he has good strong eyes. That he merely is suffering from a functional, physiological, insufficiency, which can be overcome by a short treatment of a quarter-of-an-hour each day, for a month. This does not preclude the advisability, in selected cases, of prescribing glasses suited to an abnormal refraction as well. During kinetic treatment for asthenovergence, one watches a functional unit of high perceptual level at work, viz.: convergence, the kinetic principle of bi-foveal vision. One learns to rely on it to assert itself. One can count on convergence to give the strikingly good results we have experienced, even with cruder techniques than mine. Another advantage is direct contact with what are usually keen intelligent persons, who appreciate, and try to understand what is being done for them. All this is reflected in the waiting-room, where the gloom of being measured or re-measured for glasses is replaced by a cheerful expectant attitude.

These are not the only advantages. Kinetic therapy for asthenovergence does more than merely cure eyestrain. In 1932 I have drawn attention to one remarkable result, viz.: the improvement in visual acuity in many cases ${ }^{8}$. The Lancet at the time reluctantly accepted my claim ${ }^{9}$, and called it a "startling statement." 10 I think it throws some light on the obscure problem of amblyopia. It appears that this fact has now been rediscovered ${ }^{1}$ - that treatment of eyestrain by my method in a certain number of patients improves the visual acuity. In the paper "Amblyopia"8 I stated that 25 per cent. of my eyestrain patients have-amblyopia due to asthenovergence, and that in this group the average improvement in visual acuity is 50 per cent. Williamson-Noble admits ${ }^{1}$ that such results are not uncommon, and have been achieved several times. This is a confirmation of my "startling statement" of 1932, opening up 
unexpected therapeutical vistas. This is the improvement which by Williamson-Noble is called ". . . not usually very strıking, but such results as $6 / 9$ becoming $6 / 6$ or $6 / 5$ are not uncommon, and have been achieved several times." This is respectively 50 per cent. and nearly 90 per cent. improvement. He also mentions that others have found the same thing ${ }^{1}$. It strikes one that, if after 1932 some more general attention had been paid to this matter, many thousands of keen intelligent people would have benefited meanwhile, considering that in one man's practice alone, some five hundred had their visual acuity improved this way by an average of 50 per cent.

Practical experience of kinetic therapy for astheno-vergence, as well as scientific consideration, leaves no doubt that the act of convergence is a visual reflex act, that is to say an involuntary action.

The anatomical foundation for this group of movements, as already described by me elsewhere $e^{4}$, is as follows. The unpaired nucleus, the centre of convergence ${ }^{3}$, lies across the raphe of the mid-brain. Its situation is between the paired nuclei for the recti interni, and very near the other paired oculomotor and trochlearis nuclei. These paired nuclei, together with the nuclei abducentes, are responsible for all the voluntary eye movements, binocular adduction, or voluntary squint included. Binocular adduction in man, as in the horse for instance, is actuated from the paired nuclei of the recti interni. Convergence however, that phylogenetically young acquirement of the higher mammals, is actuated by its own nucleus through the means of visual reflexes, like accommodation and the pupillary movements. Moreover, the visual reflexes that actuate convergence, can elicit vertical as well as horizontal convergence, as I have found, inter alia, in a case of squint caused by traumatic paralysis of the left trochlearis muscle after an air crash, which was treated and cured simply by kinetic treatment for astheno-vergence, modified in view of the circumstances.

Convergence has a unique place in the visual economy, and, I believe, in biology. It is, to my knowledge, the only instance where movement as such is-not function merely, but-part of a sense organ. Convergence, indeed is essential for the existence of the bifoveal double eye. It is not just a function of this double eye, hut actually part of it. Without convergence the bi-foveal double eye does not exist, but falls apart in two single foveal eyes, each perfect and complete in form and function, but antagonistic to one another, as the case of squint shows. Convergence, therefore, is the kinetic correlate, in the primates, of the binocular fovea, of the semidecussation of the optic nerves.

The solution of the problem of eyestrain has been looked for in refraction, in accommodation, in phoria, and in eikonia, in the order in which these phenomena were discovered. Refraction is the static, accommodation the kinetic visual condition of the single eye. 
Phoria and eikonia are the static visual conditions of the bi-foveal double eye, and convergence it is now clear, is the kinetic visual condition of the human double eye.

In convergence lies the main solution of the great problem of eyestrain. I have reason to belieye that squint falls in the same category. The formula for hoth disorders is to establish the full normal range of convergence. : In both, this can be done by kinetic treatment for astheno-vergence. Concomitant squint, I believe, is the consequence of infantile astheno-vergence, eyestrain the consequence of juvenile and adult astheno-vergence. Both disorders are cured by treatment of the astheno-vergence. (I need not here go into detail concerning complications in the case of squint). Traumatic squint can also be cured by kinetic treatment for astheno-vergence, as I found in two rather severe cases, one of which came under my treatment six weeks, the other three years after the accident.

There are many conditions, apart from eyestrain and squint, that benefit greatly by this kinetic treatment. Tennis players may lose some of their ability and endurance through astheno-vergence. These can be restored to full power. Air pilots, who are, or have become, indifferent or bad landers, may gain or re-gain their ability and confidence. Many patients with ophthalmic migraine can have their worst symptoms reduced, and often even eliminated. Even an alarming contraction of the visual fields, obviously the result of astheno-vergence, had its diagnosis confirmed by its prompt and lasting cure by kinetic treatment ${ }^{4}$ p. 38. More or less brilliant school children, who had lost their zest for reading or drawing, have become their old selves again after a course of kinetic treatment with the battery.

It would only be sound preventive ophthalmology for every child of school age to have his or her convergence tested, and if below the normal range of Elschnig, 50 dioptres, to have it brought to normal power by kinetic treatment with the battery of prisms.

I have treated a great number of eyestrain patients by my method. Several thousands more of such patients passed through my hands these last fifteen years, but could not, for some reason or other, he treated in the same way, amongst them thousands of children I examined in the school clinic. This emphasizes that the preventive side of present-day ophthalmology is deplorably weak.

These figures of thousands of persons, all in need of kinetic treatment, going through the hands of one ophthalmologist raises the question: what are my colleagues doing about this? It does not appear at all satisfactory that leading ophthalmologists treat a few cases, commend the results, and that we thus merely go on experimenting with this tremendous problem of eyestrain. As a result ophthalmology remains as cramped as ever, and thousands of patients in every ophthalmological practice remain "refraction cases," do not 
get further than mechanical alleviation, and drift to opticians. Preventive ophthalmology is again ignored.

Williamson-Noble's selected cases, and the cases that do. not respond well to the therapy of glasses, are subjected to a few orthoptic lessons by a trainer. I foresee that these trainers will soon become sufficiently independent to form a trade, like the opticians. They will take the "job" entirely in their own hands. And so this really great opportunity of rising above the status of opticians, and taking part in real preventive medicine will be lost to us.

$A n$ increasing number of intelligent people are getting tired of glasses where they are not absolutely necessary. " And they are indeed not absolutely necessary in the majority of our refraction cases. This applies to the greatest group, that of hypermetropia, except in a small percentage of excessive ametropia. This applies as well to most of the quarter and half dioptres of astigmatism, provided convergence has a normal range.

At present the public are still looking to us for guidance and help. They are quite prepared to pay for six hours kinetic treatment by a fully qualitied medical specialist, just as well as they are prepared to pay for an hour's refracting. If we hand them over, for occasional kinetic treatment to assistants without physiological knowledge, these assistants will soon undertake that work mechanically. I have noticed that opticians are already advertising this so-called orthoptic work. Our profession will be well advised, from the start, to keep this eminently physiological therapy out of the hands of enterprising opticians, and out of the hands of "the ladies who now, with less judgment than enthusiasm, practice orthoptics," as a London authority recently described them to me.

In the schools of ophthalmology, the time and energy spent on teaching the coming generation of ophthalmic surgeons how to refract, is not wasted, considering that refracting cases form such a high percentage of our practice. Yet, where it now has to be admitted that many of these cases are not so much in need of glasses as of treatment for astheno-vergence, the urgent need for teachers who understand, and teach physiological ophthalmology and physiological ophthalmological methods, becomes evident.

Another great problem, so far not properly dealt with, is squint, that terrible handicap in life. In 1929 I instituted an enquiry covering all the primary schools of the Transvaal, comprising about one hundred thousand children. A questionnaire was sent by the Central School Clinic. This questionnaire was accompanied by the necessary means, enabling the teachers of all the schools to examine and report on all the children, who were known, or were supposed to be, squinters. The response was satisfactory-only a few schools did not reply. The result showed that 967 children in the primary schools of the province were reported as squinters, or about 1 per 
cent. This high figure is probably an under-estimate. I have seen a similar report from a London district, where the figure was $2 \cdot 4$ per cent.

Twenty years ago, my teacher in ophthalmology made the tentative suggestion that squint might not be a matter of muscles but of brain, but nevertheless for squint he taught us to operate on the eye muscles. I confess to have done such squint operations in the earlier years of my practice only with a bad scientific conscience, thinking of the brain disorder I was operating on!

My first physiologically treated case of squint came into my hands in rather an unexpected manner. In 1932 a girl aged eight years was sent to me by her father, a surgeon, with the intimation that, as I had cured several people of eyestrain, and these people often had discarded their glasses, I might as well cure his daughter, who, for several years now, had been under ophthálmological treatment and had worn glasses for her hypermetropia without any satisfactory effect as to her squint. The girl had normal visual acuity, in either eye $6 / 6$ with and without glasses, a hypermetropia of 1.5 dioptres in both eyes, for which she was wearing the full correction. There was alternating intermittent esotropia. She was often unsquinting when looking at far objects, but not when reading or doing near work, or when she was tired. As, at the time, I had no synoptophore, diploscope or any such adjustable instrument at hand, the only thing to do seemed to treat the case kinetically, starting as high as the patient could easily take, going up and then coming down gradually to zero and negative convergence. This succeeded, even beyond expectation, as in six weeks the patient had full range of positive and negative convergence. She did not squint any more, and has normal binocular and stereoscopic vision. From the beginning of the treatment her glasses were discarded, and no glasses have been worn since.

This technique of squint treatment, adopted on the spur of the moment, I have used since, and developed for the treatment of other and more difficult cases.

One of the most difficult cases was a girl aged eight years, with $6 / 6$ vision in each eye, and an entirely neglected alternate esotropia of 35 degrees. The whole treatment lasted four years, when a cure was effected with full binocular and stereoscopic vision. I decided to take on this case because I wanted to test the verdict of textbooks that such alternating concomitant squints are precluded from ever reaching fusion or stereoscopic vision. This proved to be a fallacy.

The curability of concomitant squint by physiological means is to be beyond doubt. It should be undertaken in the primary school years. Squint should not be patched up by untimely surgical interference, but be treated as the physiological insufficiency it 
really is. Physiological treatment of all squint in school children should come within the range of possibility, if ophthalmology will rise to the occasion, and will give the lead in this great cause of preventive medicine.

\section{Summary}

Only by accepting the physiological evidence that involuntary convergence is the kinetic principle of human binocular vision, the two great problems, of Eyestrain and of Squint, can be approached correctly.

My kinetic treatment for astheno-vergence does not mean " exercises."

The difference can be understood from the text.

There is a danger of mechanizing kinetic treatment, which will eventually bring it into the hands of unqualified persons.

The paper describes the scope and the results of kinetic treatment.

There is an increasing conviction amongst the profession and the public that too much is made of correction by glasses.

The development of preventive ophthalmology is stunted through the absence of physiological insight in present-day ophthalmology.

\section{[REFERENCES}

1. Williamson-Noble, if. A. (1941).- " The Problem of Asthenopia." The Richard Middlemore Lecture, May 7. Brit. Jl. Ophthal., Vol. XXV.

2. ZEEMAN, W. P. C (1938).-Nederl. Tijdschr. v. Geneesk., January 5.

3. BROUWER, B. (1918).- "Klinische Untersuchungen ueber den Oculomotoriuskern." Zentralbl. Ges. Neurol. Psychiat., Band 40, Hft. 1/3, p. 189.

4. StutTerheim, N. A. (1937).- "Eyestrain and Convergence." London, H. K. Lewis \& Co., Ltd.

5. Elschnig, A. (1902).-" Fusion." Encyclopaedia der Augenheilkunde, p. 330 .

6. DuAnE, A. (1926).- " The Forms of Convergence," in contributions to Ophthalmic Science, dedicated to Jackson, p. 34.

7. Review (1939).-Brit. Jl. Physiol. Optics, October.

8. STUtTERHEim, N. A. (1932)'-- "Amblyopia." Lancet, pp. 222, 774.

9. AnNOTATION (1932).-Lancet, pp. 222, 789.

10. Stutterheim, N. A. (1932).--Lancet, pp. 222, 1386.

\section{ANNOTATION}

\section{Paper Salvage}

Our readers will probably have noticed the slight difference in texture and tint of the paper used for printing the Journal since the beginning of this year. Our stock of paper came to an end in December last and like everybody else we have had to make do with what the controller allows us. In after years it may be of interest as dating some of the War years. Deterioration in 